\title{
Internationale Vergleiche von Kosten und Qualität
}

\author{
Die Kostenunterschiede zwischen den nationalen Gesundheitswesen sind erheblich, \\ auch zwischen gut vergleichbaren Ländern. Gemäss drei verschiedenen Ranglisten \\ ist die Schweiz das drittteuerste Land, mit jährlichen Ausgabenzunahmen von 2,4 bis \\ 2,9 Milliarden Franken. Die Direktbelastung der Kranken ist bei uns am höchsten. \\ Zahlen aus der neuesten OECD-CD werden ergänzt durch Qualitätsranglisten von \\ fünf anderen Organisationen.
}

«Round up the usual suspects», befiehlt Polizeichef Renault im Kultfilm Casablanca von 1942. Wendet man diese Taktik auf die Fahndung nach den Ländern mit den höchsten Gesundheitsausgaben an, wird man rasch fündig. Bei den Pro-Kopf-Gesundheitsausgaben 2008 sind die Spitzenreiter die USA, Norwegen und die Schweiz, genau gleich wie schon 2007 und 2006. Dies gilt sowohl für die Ausgaben zum Wechselkurs wie auch kaufkraftbereinigt.

Diesen internationalen Vergleich kann man der neuesten Ausgabe der CD der Organisation für wirtschaftliche Zusammenarbeit und Entwicklung entnehmen: OECD Health Data 2010 [1]*. Wie jedes Jahr enthält auch diese 19. Auflage über 1200 Indikatoren und Zahlenreihen über die nationalen Gesundheitswesen der 32 OECD-Länder sowie neu von Estland und Israel (beide wurden kürzlich zur Mitgliedschaft eingeladen). Die rund 790000 Daten betreffen 2008 und teilweise 2009, aber oft sind Zahlen nur bis 2007 vorhanden. Die Abdeckung nimmt von Jahr zu Jahr zu. Leider gibt es aber in den Zahlenreihen vieler Länder - gerade auch der Schweiz - immer noch zahlreiche weisse Stellen. Gleichwohl ist die OECD-CD für internationale Vergleiche praktisch unentbehrlich.

\section{Grosse Unterschiede}

\section{bei den Gesundheitsausgaben pro Kopf}

Tabelle 1 zeigt die Ausgaben kaufkraftbereinigt, also unter Berücksichtigung der unterschiedlichen Kaufkraft in den verschiedenen Ländern. Mit 7538 USD pro Einwohner geben die Amerikaner fast zweieinhalbmal mehr für ihr Gesundheitssystem aus als der Durchschnitt der OECD-Länder. Verglichen mit der drittplazierten Schweiz (4627 USD) sind die US-Ausgaben immer noch um $63 \%$ höher. Die Schweiz gibt $50 \%$ mehr aus als der Durchschnitt der OECD-Länder. Von 2000 bis 2008 nahmen die Pro-Kopf-Ausgaben in allen OECD-Ländern zu, im Schnitt um 69\%. Mit 60 bis $65 \%$ knapp darunter lagen Norwegen, Kanada, Finnland und die USA. Um 40 bis $45 \%$ stiegen die Ausgaben in Frankreich, der Schweiz, Österreich, Deutschland und Italien.

\section{Tabelle 1}

Gesundheitsausgaben pro Kopf in US-Dollar (kaufkraftbereinigt) 2008 (teilweise 2007).

\begin{tabular}{|c|c|c|c|}
\hline Land & USD & $\begin{array}{l}\text { Index: } \\
\text { Durchschnitt aller } \\
\text { Länder }=100\end{array}$ & $\begin{array}{l}\text { Zunahme } \\
\text { in } \% \\
\text { seit } 2000\end{array}$ \\
\hline USA & 7538 & 244 & 60 \\
\hline Norwegen & 5003 & 162 & 65 \\
\hline Schweiz & 4627 & 150 & 44 \\
\hline Kanada & 4079 & 132 & 62 \\
\hline Niederlande & 4063 & 132 & 74 \\
\hline Österreich & 3970 & 129 & 41 \\
\hline Irland & 3793 & 123 & 110 \\
\hline Deutschland & 3737 & 121 & 40 \\
\hline Frankreich & 3696 & 120 & 45 \\
\hline Belgien & 3677 & 119 & 55 \\
\hline Dänemark* & 3540 & 115 & 49 \\
\hline Schweden & 3470 & 112 & 52 \\
\hline Island & 3359 & 109 & 23 \\
\hline Australien* & 3353 & 109 & 48 \\
\hline Grossbritannien & 3129 & 101 & 71 \\
\hline Finnland & 3008 & 97 & 62 \\
\hline Spanien & 2902 & 94 & 89 \\
\hline Italien & 2870 & 93 & 40 \\
\hline Japan* & 2729 & 88 & 39 \\
\hline Griechenland* & 2687 & 87 & 85 \\
\hline Neuseeland & 2683 & 87 & 67 \\
\hline Slowenien & 2329 & 75 & 60 \\
\hline Israel & 2244 & 73 & 23 \\
\hline Südkorea & 1801 & 58 & 123 \\
\hline Tschechien & 1781 & 58 & 82 \\
\hline Slowakei & 1738 & 56 & 188 \\
\hline Ungarn & 1437 & 47 & 69 \\
\hline Estland & 1263 & 41 & 142 \\
\hline Polen & 1213 & 39 & 106 \\
\hline Mexiko & 852 & 28 & 68 \\
\hline Durchschnitt & 3086 & 100 & 69 \\
\hline
\end{tabular}

Korrespondenz:

Dr. rer. pol. Gerhard Kocher Haldenweg $10 \mathrm{~A}$ $\mathrm{CH}-3074$ Muri gerhard.kocher@muri-be.ch
Die Literaturangaben finden www.saez.ch $\rightarrow$ Aktuelle Nummer oder $\rightarrow$ Archiv 
Ein ähnliches Bild ergibt Tabelle 2 mit den ProKopf-Ausgaben zu Wechselkursen. Hier liegt Norwegen mit dem 2,2Fachen des OECD-Durchschnittes an oberster Stelle. Nach den USA ist die Schweiz mit 7037 USD wiederum im dritten Rang: Sie gibt 92\% mehr aus als der OECD-Schnitt. In allen drei Ländern lag die Ausgabenzunahme zwischen 2000 und 2008 unter dem OECD-Durchschnitt von 165\%.

Ein Vergleich mit anderen Weltregionen ist deprimierend: Für Südostasien verzeichnet die WHO (2007) jährliche Gesundheitsausgaben von 41 USD pro Kopf, für Afrika 76 USD [2]. Die Extreme sind 7 USD in Burma und 7439 USD in den USA - ein Verhältnis von 1:1063.

\section{Tabelle 2}

Gesundheitsausgaben pro Kopf in USD, (zu Wechselkursen), 2008 (teilweise 2007).

\begin{tabular}{|c|c|c|c|}
\hline Land & USD & $\begin{array}{l}\text { Index: } \\
\text { Durchschnitt aller } \\
\text { Länder }=100\end{array}$ & $\begin{array}{l}\text { Zunahme } \\
\text { in } \% \\
\text { seit } 2000\end{array}$ \\
\hline Norwegen & 8075 & 221 & 156 \\
\hline USA & 7538 & 206 & 60 \\
\hline Schweiz & 7037 & 192 & 99 \\
\hline Dänemark* & 5550 & 152 & 124 \\
\hline Irland & 5500 & 150 & 253 \\
\hline Niederlande & 5274 & 144 & 175 \\
\hline Österreich & 5215 & 142 & 121 \\
\hline Frankreich & 4996 & 136 & 127 \\
\hline Belgien & 4932 & 135 & 143 \\
\hline Schweden & 4879 & 133 & 114 \\
\hline Island & 4777 & 130 & 63 \\
\hline Deutschland & 4714 & 129 & 99 \\
\hline Kanada & 4691 & 128 & 125 \\
\hline Finnland & 4282 & 117 & 153 \\
\hline Australien* & 3975 & 109 & 130 \\
\hline Grossbritannien & 3838 & 105 & 117 \\
\hline Italien & 3545 & 97 & 129 \\
\hline Spanien & 3240 & 88 & 213 \\
\hline Neuseeland & 2953 & 81 & 180 \\
\hline Japan* & 2781 & 76 & -2 \\
\hline Griechenland* & 2679 & 73 & 192 \\
\hline Slowenien & 2257 & 62 & 172 \\
\hline Israel & 2222 & 61 & 44 \\
\hline Tschechien & 1498 & 41 & 315 \\
\hline Slowakei & 1374 & 38 & 564 \\
\hline Südkorea & 1245 & 34 & 129 \\
\hline Ungarn & 1119 & 31 & 243 \\
\hline Estland & 1073 & 29 & 389 \\
\hline Polen & 972 & 27 & 294 \\
\hline Chile & 697 & 19 & 115 \\
\hline Mexiko & 599 & 16 & 83 \\
\hline Durchschnitt & 3662 & 100 & 165 \\
\hline
\end{tabular}

\section{Schweiz: massive Mehrausgaben}

\section{trotz aller Sparmassnahmen}

Die OECD schätzt die schweizerischen Gesundheitsausgaben 2008 auf 58,1 Mrd. CHF, 2009 auf 60,6 Mrd. CHF. Zwischen 2007 und 2009 gaben wir jährlich 2,4 bis 2,9 Mrd. CHF mehr aus als im Vorjahr. Dies entspricht einer Zunahme von 4,5 bis 5,3\% pro Jahr. Trotz bald flächendeckender Klagen über die Sparpolitik - wenn nicht gar über eine «Sparhysterie» - sind die Gesundheitskosten also weit davon entfernt, unter Kontrolle zu sein, wie ein häufig genanntes Ziel lautet. Faktisch heisst in der Gesundheitspolitik «sparen»: mehr ausgeben.

Eine KOF-Prognose [3] schätzt übrigens die Gesamtausgaben für 2009 auf 61 Mrd. CHF (+5,3 Prozent), für 2010 auf 63,3 Mrd. (+5 Prozent) und für 2011 auf 65,6 Mrd. CHF (+ 3,7 Prozent).

Die Bevölkerungszunahme ist am Ausgabenwachstum nur marginal schuld: Selbst die rekordhohe Zunahme um 108000 Einwohner im Jahr 2008 erklärt rechnerisch nur 1,4\% der Kostenzunahme in diesem Jahr (41 Millionen von 2,9 Mrd. CHF). Länder mit einem hohen Bevölkerungsanteil Älterer haben nicht notwendigerweise hohe Gesundheitsausgaben. In Japan machen die über 79-Jährigen 5,9 Prozent der Bevölkerung aus, in den USA nur 3,8 Prozent (über 64-Jährige: Japan 22,1; USA 12,7 Prozent). Die japanischen Gesundheitsausgaben betragen aber nur rund $36 \%$ der US-Ausgaben (Tab. 1 und 2).

\section{Gesundheitsausgaben im Verhältnis zum Bruttoinlandprodukt}

Internationale Kostenvergleiche in absoluten Dollaroder Franken-Beträgen müssen ergänzt werden durch die Relationen zum nationalen Bruttoinlandprodukt BIP (Tab. 3). Es ist dies ein Indikator für den Wohlstand im betreffenden Land und für die aktuelle Wirtschaftslage. Die Spitzenreiter sind einmal mehr die USA im ersten und die Schweiz im dritten Rang mit 10,7\% des BIP. Im zweiten Rang ist neu Frankreich, während Norwegen sich nur noch auf dem 18. Rang findet. Der Grund liegt vor allem darin, dass Norwegen dank Öl und Gas 2008 ein extrem hohes Pro-KopfBruttoinlandprodukt (BIP) erzielte: 59000 Dollar gegenüber 47000 der USA und 42000 der Schweiz.

\section{Tabelle 3}

Gesundheitsausgaben pro Kopf in \% des Bruttoinlandprodukts BIP, 2008 (teilweise 2007).

\begin{tabular}{|c|c|c|c|}
\hline Land & USD & $\begin{array}{l}\text { Index: } \\
\text { Durchschnitt aller } \\
\text { Länder }=100\end{array}$ & $\begin{array}{l}\text { Zunahme } \\
\text { in } \% \\
\text { seit } 2000\end{array}$ \\
\hline USA & 16 & 178 & 18 \\
\hline Frankreich & 11,2 & 124 & 11 \\
\hline Schweiz & 10,7 & 119 & 5 \\
\hline Deutschland & 10,5 & 117 & 2 \\
\hline Österreich & 10,5 & 117 & 6 \\
\hline
\end{tabular}




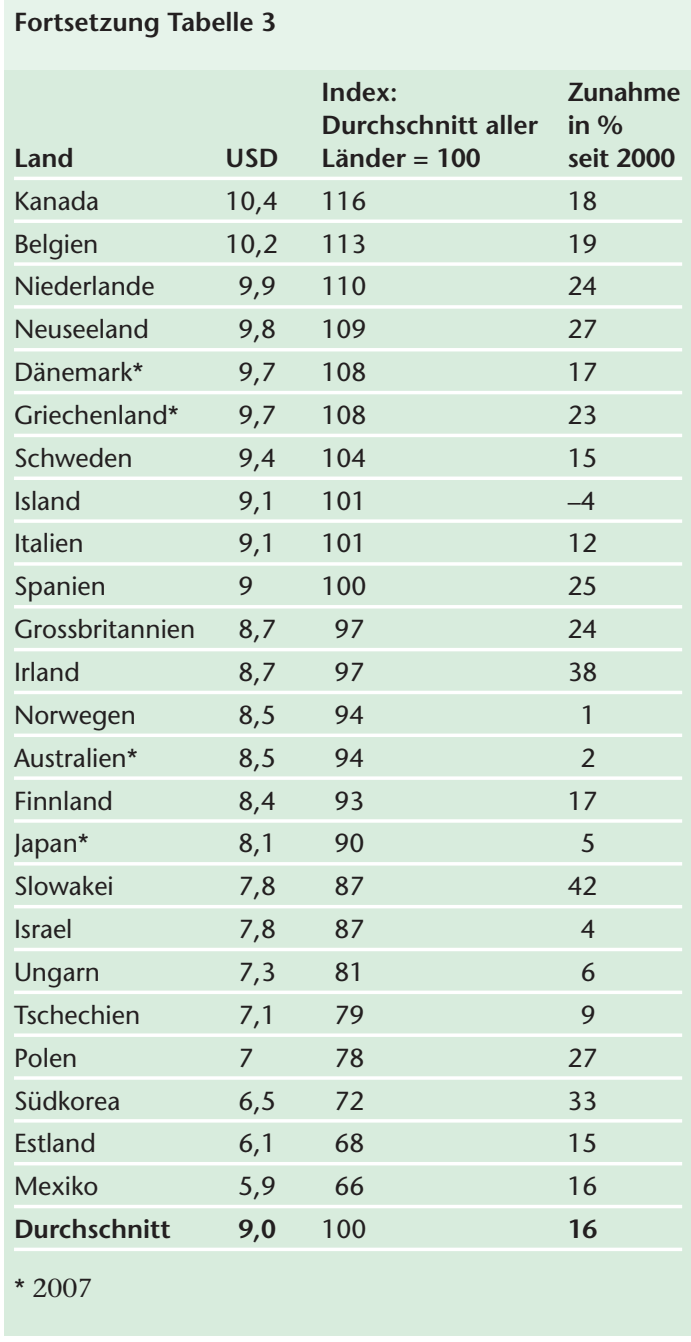

Der enge Zusammenhang zwischen der Höhe des BIP und den Gesundheitsausgaben ist seit langem bekannt und wird auch in Abbildung 1 deutlich. Sie zeigt das BIP und die Gesundheitsausgaben, beide pro

Abbildung 1

Gesundheitsausgaben und Bruttoinlandprodukt, 2008, pro Kopf, in USD (kaufkraftbereinigt).

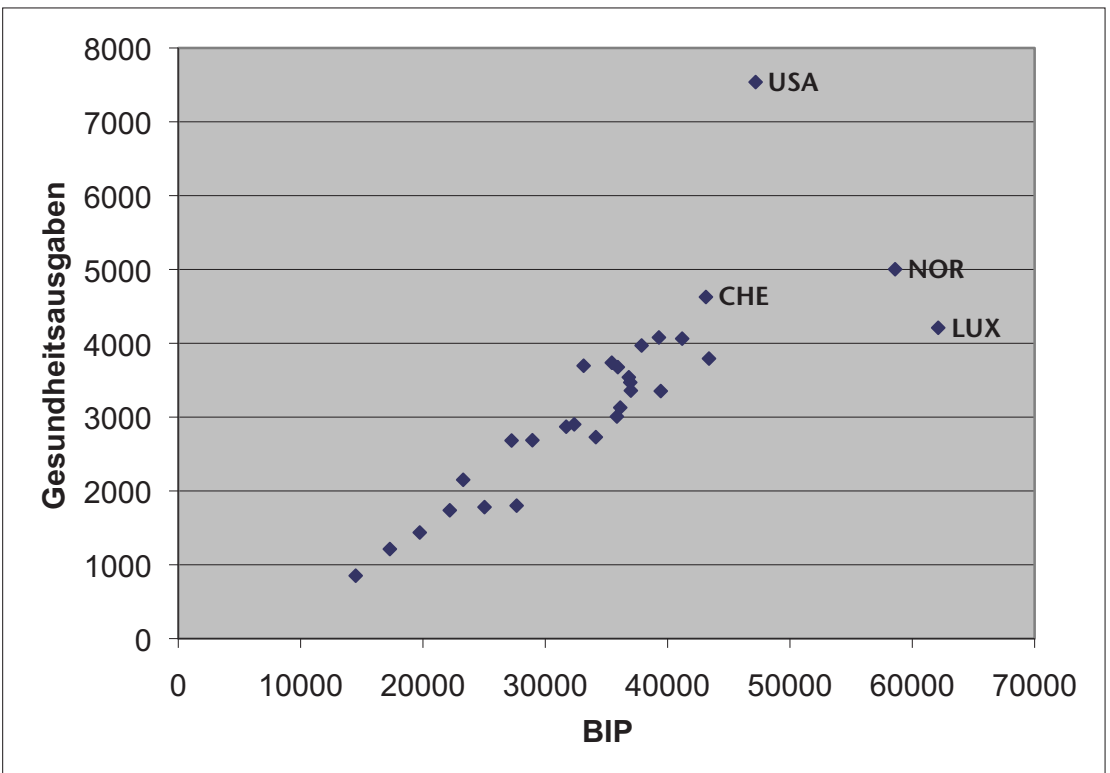

Einwohner. Der extreme Ausreisser sind einmal mehr die USA. Die beiden anderen Ausreisser Luxemburg und Norwegen haben ein markant höheres BIP pro Kopf (32 bzw. 24 Prozent höher) als die USA. Ihre Gesundheitsausgaben sind aber um 44, resp. 34\% tiefer als die der USA. Das Schweizer BIP/Kopf ist gegenüber den USA $9 \%$ tiefer, die Gesundheitsausgaben sind 38 Prozent tiefer.

\section{Gesundheitsausgaben im Verhältnis zum empfundenen Gesundheitszustand}

Fühlen sich die Leute in Ländern mit hohen Gesundheitsausgaben gesünder? Die Abbildung 2 vergleicht die Pro-Kopf-Ausgaben in US-Dollar (kaufkraftbereinigt) mit dem subjektiv empfundenen Gesundheitszustand, gemessen in Prozent der Bevölkerung, die ihn als «gut» bezeichnen. Die USA erzielen hier eine hohe Zufriedenheit und den dritten Rang von 24 Ländern. Allerdings fühlen sich im gleichen Cluster A die Neuseeländer und die Kanadier noch gesünder, obwohl sie pro Kopf 64, resp. $46 \%$ weniger als die Amerikaner ausgeben. Die Schweiz erzielt mit 39\% tieferen Ausgaben fast die gleiche hohe subjektive Zufriedenheit mit dem Gesundheitszustand wie die USA.

Cluster B besteht aus Norwegen, den Niederlanden und Island. Der fast gleiche Gesundheitszustand wird in Norwegen und Island registriert, obwohl Norwegen 5003 und Island nur 3359 USD pro Kopf für ihr Gesundheitssystem ausgeben. Mit ähnlich hohen Ausgaben variiert im Cluster C der subjektive Gesundheitszustand zwischen Deutschland, Frankreich, Belgien und Irland enorm - nur 64,7\% «gut» in Deutschland, 72,4\% in Frankreich, 76,7\% in Belgien und 84,4 in Irland. Noch grössere Diskrepanzen zeigt trotz vergleichbarer Gesundheitsausgaben der Cluster D mit der Slowakei, Südkorea und Tschechien.

Ein teilweise ganz anderes Bild ergibt eine Meinungsbefragung in sechs Ländern [4]. Ihren Gesundheitszustand benoten 61 Prozent der Befragten in Kanada als «ausgezeichnet oder sehr gut», gefolgt von den USA (60), der Schweiz (51), und Grossbritannien (49). Weit abgeschlagen sind Frankreich mit 31 Prozent und Deutschland mit nur 23 Prozent.

\section{Schweiz: höchste Direktbelastung der Kranken}

Einsame Spitze ist die Schweiz weiterhin bei den «Selbstzahlungen der Privathaushalte», also bei den Gesundheitsleistungen, die direkt aus dem eigenen Portemonnaie bezahlt werden müssen (Tab. 4). Diese Out-of-pocket-Zahlungen betreffen vor allem Leistungen für Pflegeheime, Zahnmedizin, Institutionen für Behinderte und rezeptfreie Medikamente. Wir geben dafür 2166 USD pro Kopf aus, der Durchschnitt der erfassten Länder nicht einmal ein Drittel davon (599 USD). Die am zweitstärksten belasteten Norweger zahlen nur etwas mehr als die Hälfte der Schweizer.

Dazu kommt, dass die Kostenbeteiligungen der Versicherten in der Schweiz ebenfalls hoch sind. Die 
Abbildung 2

Gesundheitsausgaben und subjektiver Gesundheitszustand, 2008 (teilweise 2007).

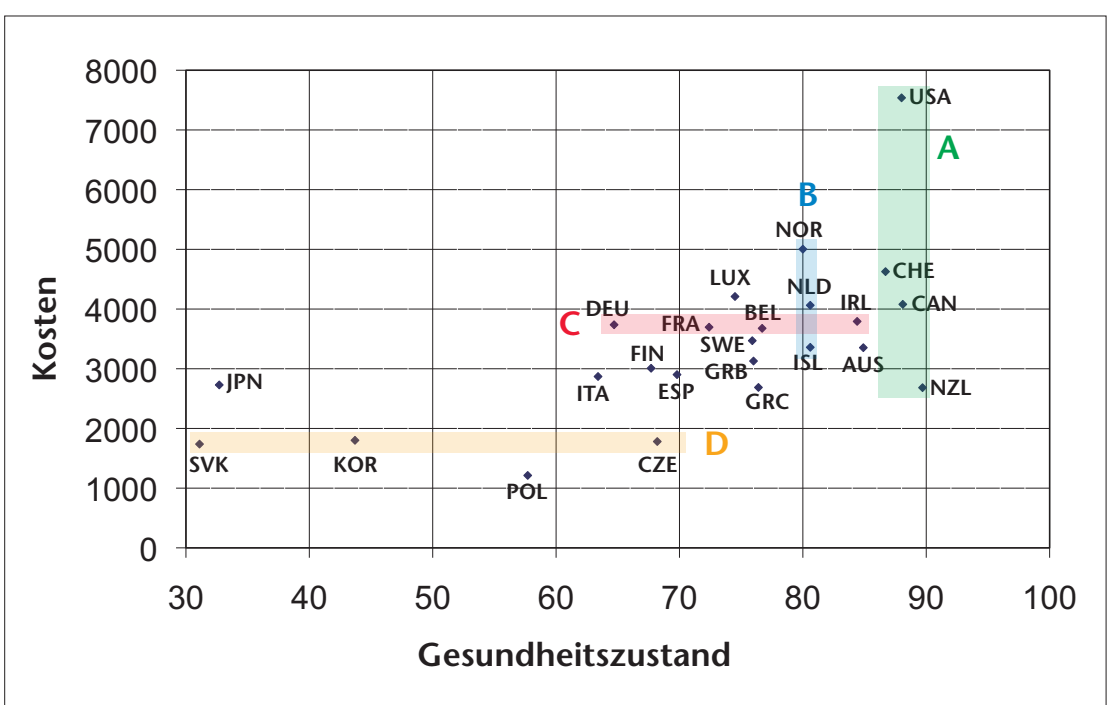

\section{Tabelle 4}

Selbstzahlungen der Privathaushalte 2008, in USD pro Kopf (Wechselkurs) und in Prozent des Bruttoinlandprodukts.

\begin{tabular}{|c|c|c|c|c|}
\hline Land & US-\$ & $\begin{array}{l}\text { Zu-/Abnahme } \\
\text { in \% seit } 2000\end{array}$ & \% BIP & $\begin{array}{l}\text { Zu-/Abnahme } \\
\text { in \% seit } 2000\end{array}$ \\
\hline Schweiz & 2166 & 86 & 3,3 & 0 \\
\hline Norwegen & 1219 & 131 & 1,3 & -7 \\
\hline USA & 912 & 34 & 1,9 & 0 \\
\hline Finnland & 831 & 120 & 1,6 & 0 \\
\hline Irland & 792 & 234 & 1,3 & 44 \\
\hline Österreich & 788 & 118 & 1,6 & 7 \\
\hline Dänemark* & 766 & 93 & 1,3 & 0 \\
\hline Island & 732 & 31 & 1,4 & -22 \\
\hline Italien & 690 & 82 & 1,8 & -10 \\
\hline Kanada & 690 & 108 & 1,5 & 7 \\
\hline Spanien & 672 & 175 & 1,9 & 12 \\
\hline Israel & 661 & 78 & 2,3 & 28 \\
\hline Deutschland & 615 & 135 & 1,4 & 27 \\
\hline Südkorea & 436 & 77 & 2,3 & 5 \\
\hline Grossbritannien & 425 & 79 & 1,0 & 11 \\
\hline Neuseeland & 410 & 153 & 1,4 & 17 \\
\hline Japan* & 406 & -15 & 1,2 & -8 \\
\hline Frankreich & 370 & 137 & 0,8 & 14 \\
\hline Slowakei & 353 & 1505 & 2,0 & 233 \\
\hline Niederlande & 303 & 76 & 0,6 & -14 \\
\hline Mexiko & 295 & 77 & 2,9 & 12 \\
\hline Ungarn & 267 & 210 & 1,7 & -6 \\
\hline Tschechien & 236 & 574 & 1,1 & 83 \\
\hline Polen & 218 & 195 & 1,6 & -6 \\
\hline Estland & 211 & 380 & 1,2 & 9 \\
\hline Türkei* & 116 & 115 & 1,3 & -7 \\
\hline Durchschnitt & 599 & 192 & 1,6 & 17 \\
\hline
\end{tabular}

Statistik der obligatorischen Krankenversicherung 2008 [5] schätzt diese Ausgaben auf 3,3 Milliarden CHF. Die Direktbelastung der Patienten in der Krankenversicherung steigt seit Jahren stärker als die Gesundheitskosten und nimmt seit 2008 noch mehr zu. Je

\section{Die Behandlungskosten werden weniger durch die Kranken als durch das Angebot und die Behandler bestimmt}

nach Ideologie und Menschenbild wird die extrem hohe Direktbelastung als Eigenverantwortung gelobt oder als Bestrafung der Kranken kritisiert. Zu wenig bekannt ist die folgende Tatsache: Eine niedrige Selbstbeteiligung ist zwar sozial vertretbar, medizinisch verantwortbar und politisch durchsetzbar, nützt aber nichts. Eine hohe Selbstbeteiligung würde zwar nützen, ist aber unsozial, medizinisch gefährlich und politisch nicht durchsetzbar.

Noch wichtiger ist die grundlegende Erkenntnis der Gesundheitsökonomie, dass die Behandlungskosten weniger durch die Kranken als durch das Angebot und die Behandler bestimmt werden (Art und Dauer der Behandlung, Einweisung, Verschreibung usw.). Wer im Gesundheitswesen sparen will, muss in erster Linie auf das Angebot und die Anbieter und weniger auf die Nachfrage einwirken. Struktur- und Behandlerbeeinflussung bringt mehr als Patientenbestrafung.

\section{OECD-Kerndaten zu den Krankenhäusern}

Dieser jährliche Überblick in der Schweizerischen Ärztezeitung über die neuesten OECD-Daten ist platzmässig beschränkt. Verschiedene Themen wechseln deshalb von Jahr zu Jahr. Die Akutbettenbelegung wurde letztes Jahr dargestellt [6], die Bettendichte und die Krankenhaustage pro Kopf im Jahr 2008 [7]. Tabelle 5 vergleicht wichtige Krankenhausdaten von sieben Ländern. Danach hatte unser Land 2008 die längste Aufenthaltsdauer, die höchste Bettenbelegung und am meisten Krankenhauspersonal pro 1000 Einwohner, gemessen in Vollzeitäquivalenten. Bezüglich Personaldichte gemessen in Stellen war die Schweiz im zweiten Rang. Die Zahl der Krankenhaustage pro Einwohner und Jahr lag genau im 7-Länder-Durchschnitt, die Bettendichte war unterdurchschnittlich.

In der Schweiz stiegen laut OECD von 2000 bis 2008 der Personalbestand um 13\%, die Vollzeitäquivalente um $11 \%$ und die Bettenbelegung um $4 \%$. Andererseits sanken die Bettendichte und die Aufenthaltsdauer um 17\% und die Krankenhaustage pro Kopf und Jahr um $15 \%$. Dies sind Entwicklungen von grösster Bedeutung für die Patienten, die Pflegenden und Mediziner, die Spitäler, die Versicherer und die kantonalen Finanzen. Zusammen mit den Fallpauschalen verändern sie den Krankenhaussektor massiv. 
Tabelle 5

Krankenhausdaten von 7 Ländern, 2008.

\begin{tabular}{|c|c|c|c|c|c|c|}
\hline Land & $\begin{array}{l}\text { Personalbestand } \\
\text { (Stellen) pro } \\
1000 \text { Einwohner }\end{array}$ & $\begin{array}{l}\text { Personalbestand } \\
\text { (Vollzeitäquivalente) } \\
\text { pro } 1000 \text { Einwohner }\end{array}$ & $\begin{array}{l}\text { Krankenhausbetten*/ } \\
1000 \text { Einwohner }\end{array}$ & $\begin{array}{l}\text { Krankenhaustage* / } \\
\text { Einwohner }\end{array}$ & $\begin{array}{l}\text { Bettenbelegung* } \\
\text { in } \%\end{array}$ & $\begin{array}{l}\text { Aufenthaltsdauer*, } \\
\text { Tage }\end{array}$ \\
\hline Deutschland & 14,7 & 10,8 & 5,7 & 1,6 & 76,2 & 7,6 \\
\hline Frankreich & 19,5 & 16,8 & 3,5 & 1 & 74,2 & 5,2 \\
\hline Grossbritannien & 24,6 & $\ldots$ & 2,7 & 0,8 & 84,5 & 7,1 \\
\hline Niederlande & 15,8 & 11,1 & 2,9 & 0,7 & 55,7 & 5,9 \\
\hline Österreich & $\ldots$ & 15,6 & 5,6 & 1,8 & 80,6 & 6,8 \\
\hline Schweiz & 23,3 & 17,4 & 3,3 & 1,1 & 88,2 & 7,7 \\
\hline USA & $\ldots$ & 17 & $\ldots$ & 0,6 & 66,4 & 5,5 \\
\hline Durchschnitt & 19,6 & 14,8 & 4,0 & 1,1 & 75,1 & 6,5 \\
\hline Rang der Schweiz & 2 & 1 & 4 & 3 & 1 & 1 \\
\hline
\end{tabular}

\section{Bestes Gesundheitswesen?}

Bei der Durchsicht dieser Ländervergleiche kann die Frage aufkommen, welches Land denn nun das beste Gesundheitssystem hat. Niemand geringerer als die WHO hat vor zehn Jahren kühn versucht, diese Frage in ihrem «World Health Report 2000» zu beantworten [8]. 191 Länder wurden für 1997 nach fünf Faktorengruppen benotet. Die Rangliste der ersten zehn bezüglich «Overall health system attainment»: Japan, Schweiz, Norwegen, Schweden, Luxemburg, Frankreich, Kanada, Niederlande, Grossbritannien, Österreich. Deutschland erreichte Rang 14, die USA Rang 15.

Eine zweite Rangliste in diesem WHO-Bericht bewertete die Leistung im Verhältnis zum Resultat, welches dieses Land mit seinen Ressourcen theoretisch hätte erreichen können. Hier war die Rangliste höchst überraschend: Die ersten zehn waren Frankreich, Italien, San Marino, Andorra, Malta, Singapur, Spanien, Oman, Österreich und Japan. Weitere Ränge: Niederlande 17, Grossbritannien 18, Schweiz 20, Schweden 23, Deutschland 25, Kanada 30 und die USA 37. Vor allem diese zweite Rangliste stiess auf grösste Kritik, so dass die WHO seither auf solche internationale Qualitätsvergleiche ganzer Gesundheitswesen verzichtet.

Nicht so andere Organisationen. So hat im Juni der Commonwealth Fund, eine angesehene private US-Stiftung, einen 7-Länder-Vergleich publiziert [9]. Detailliert bewertet werden die Qualität (effektiv, sicher, koordiniert und patientenorientiert), der Zugang (finanzielle Hindernisse, Rechtzeitigkeit) sowie Effizienz, Gleichheit und die Lebenserwartung (mit drei Kriterien). Die Rangliste 2010: Niederlande, Grossbritannien, Australien, Deutschland, Neuseeland, Kanada, USA. Die beiden Länder mit den höchsten Gesundheitsausgaben kommen also über die Schlussplätze nicht hinaus.

Im bereits zitierten Deloitte-Bericht [4] über sechs Länder bewerteten die Befragten die Gesamtleistung (overall performance) ihres Gesundheitswesens: Frankreich und die Schweiz kommen auf je 55 Prozent «ausgezeichnet oder sehr gut», Kanada auf 43,
Grossbritannien auf 30, die USA auf 21 und Schlusslicht Deutschland auf 17 Prozent.

In einer 16-Länder-Studie (ohne die Schweiz) der Accenture [10] siegte Singapur bei der Frage nach der Qualität der Versorgung: 87 Prozent der Befragten bewerteten sie als sehr oder ziemlich gut. Es folgten Frankreich (81), Grossbritannien (79), Kanada (78), Australien (69), Norwegen (68) und Spanien (58 Prozent). Deutschland erreichte den 8 . Rang (56 Prozent), die USA den 9. Rang mit 55 Prozent. Grosses oder ziemlich grosses Vertrauen in die Behörden, die Qualität der Versorgung zu verbessern, variierte von nur 30 Prozent der Befragten (Italien) bis zu 86 Prozent (Singapur). Deutschland war mit 34 Prozent im zweitletzten Rang.

Eine Umfrage in acht Ländern [11] zum nationalen Gesundheitswesen ergab einen klaren Sieger: In Österreich stuften 86 Prozent der Befragten die Organisation ihres Gesundheitswesens als ausgezeichnet, sehr gut oder gut ein. Die weiteren Ränge: England 70, Schweden 69, Frankreich 61, Deutschland 57, USA 44, Italien 40 und Polen 20 Prozent.

Schliesslich ist auf den Euro-Canada Health Consumer Index 2010 hinzuweisen [12]. Er vergleicht 33 europäische Länder und Kanada nach 32 patientenbezogenen Kriterien. Die Hauptbereiche sind Patientenrechte und -information (10 Kriterien), Wartezeiten für Behandlungen (5), Outcome (7), Umfang und Erreichbarkeit der Gesundheitsdienste (6) sowie Medikamente (4 Kriterien). Die Rangfolge der ersten zehn Länder: Niederlande (857 Punkte), Deutschland, Island, Frankreich, Schweiz (806 Punkte), Österreich, Dänemark, Luxemburg, Schweden und Belgien. Italien ist im Rang 14, Grossbritannien im Rang 17.

Solche Ranglisten [13] erlauben kein abschliessendes Gesamturteil über die Qualität der verschiedenen Gesundheitswesen. Immerhin ist frappant, dass die USA mit ihren extrem hohen Gesundheitsausgaben keine besseren Ränge erreichen. Die Schweiz mit den dritthöchsten Ausgaben dagegen kommt weit besser weg - auch wenn sie in jeder Rangliste von sparsameren Ländern geschlagen wird. 


\section{Literatur}

1 OECD Health Data 2010 on CD-ROM, Statistics and Indicators. Paris: OECD Publishing; 2010. Englisch/ französisch/deutsch/spanisch. 85 EUR. ISBN 978-92-64-08607-4. www.oecd.org/health/ healthdata. Siehe auch: Gesundheit auf einen Blick 2009. Paris: OECD Publishing; 2010. ISBN 978-92-64-08049-2.

2 World Health Statistics 2010. WHO. Genf; 2010. Volltext: www.who.int/whosis/whostat/ EN_WHS10_Full.pdf.

3 Konjunkturforschungsstelle ETH Zürich KOF, Mai 2010. www.kof.ethz.ch

42010 Global Survey of Health Care Consumers Behaviours, attitudes and unmet needs. Washington/London: Deloitte; 2010. www.deloitte. com/assets/Dcom-UnitedStates/Local\%20Assets/ Documents/us_chs_consumerism_Global.pdf

5 Bundesamt für Gesundheit. Bern; 2010.

6 Kocher G. Schweiz: dritthöchste Gesundheitsausgaben im neuesten OECD-Vergleich. Schweiz Ärztezeitung. 2009;90(46):1806-11.

7 Kocher G. Unser Gesundheitswesen im internationalen Vergleich. Schweiz Ärztezeitung. 2008;89(44):1911-16.
8 The World Health Report 2000: Health systems: Improving performance. WHO. Genf; 2000. Volltext: www.who.int/whr/2000/en/whr00_en.pdf.- Kritisch dazu u. a.: Whitman G. WHO's Fooling Who? Washington D.C: Cato Institute; 2008. www.cato org.- Møller Pedersen K. The World Health Report 2000: Dialogue of the deaf? Health Economics. 2002;11:93-101.

9 Mirror, mirror on the wall. How the performance of the U.S. health care system compares internationally, 2010 update. New York/Washington D.C: Commonwealth Fund; 2010. Volltext: www.commonwealthfund.org.

10 Measuring People's Impressions of Health Care. Accenture, 2010. www.accenture.com

11 Baromètre Cercle Santé, Europ Assistance, 21.9.2010 www.europe-assistance.com

12 Euro-Canada Health Consumer Index 2010. Health Consumer Powerhouse, Brüssel und Stockholm/ Frontier Centre for Public Policy. Winnipeg; 2010. Volltext: www.fcpp.org/files/1/10-05-10-Euro-Canada_Index_2010_FINAL.pdf

13 Weitere finden sich in: Kocher G, Oggier W (Hrsg.) Gesundheitswesen Schweiz 2010-2012. Bern: Hans Huber; 2010. ISBN 978-3-456-84803-7.- Version française: Kocher G, Oggier W (éds.). Système de santé suisse 2010-2012. Berne: Hans Huber; 2010 ISBN 978-3-456-84888-4. 